\title{
Concurrent Primary Gastric and Pulmonary Diffuse Large B-Cell Lymphoma: A Case Report and Literature Review
}

\author{
Ahmed Dirweesh ${ }^{\mathrm{a}, \mathrm{d}}$, Sumera Bukharia ${ }^{\mathrm{a}}$ Chikezie Alvarez ${ }^{\mathrm{a}}$, Muhammad Khan ${ }^{\mathrm{a}}$, \\ Cheryl Rimmer ${ }^{\mathrm{b}}$, Robert Shmuts ${ }^{\mathrm{c}}$
}

\begin{abstract}
The primary pulmonary diffuse large B-cell lymphoma (PPDLBCL), a kind of non-Hodgkin's lymphomas, itself is a rare entity. Its association with primary B-cell gastric lymphoma has not been established yet. Herein we present a case of PPDLBCL along with a literature review. This case is special and extremely rare as it has concurrent primary gastric B-cell lymphoma.
\end{abstract}

Keywords: Stomach; Gastric lymphoma; Pulmonary lymphoma

\section{Introduction}

The gastrointestinal (GI) tract is the most common extranodal site for lymphoma, accounting for $15-20 \%$ of all cases of extranodal lymphoma [1]. Of the GI tract, the most common sites of involvement include the stomach, followed by the small intestine and ileocecal region. Symptomatic presentation is often similar to other malignancies including weight loss, early satiety, nausea, vomiting, and anorexia, and some patients may also experience occult GI bleeding.

Primary pulmonary lymphoma (PPL) is an exceedingly rare disease that comprises $<0.5 \%$ of all primary lung tumors [2]. Most (70-90\%) PPLs are a low-grade mucosa-associated lymphoid tissue (MALT) type. The primary pulmonary diffuse large B-cell lymphoma (PPDLBCL) is particularly rare and accounts for only $10 \%$ of cases of primary pulmonary nonHodgkin's lymphoma (NHL) [3]. However, due to its rapid

Manuscript submitted May 13, 2017, accepted June 12, 2017

aDepartment of Internal Medicine, Seton Hall University-Hackensack Meridian School of Medicine, Saint Francis Medical Center, Trenton, NJ, USA

bepartment of Pathology, Our Lady of Lourdes Hospital, Willingboro, NJ, USA

'Department of Gastroenterology, Our Lady of Lourdes Hospital, Willingboro, NJ, USA

${ }^{\mathrm{d} C}$ Corresponding Author: Ahmed Dirweesh, Department of Internal Medicine, Seton Hall University, Saint Francis Medical Center, Trenton, NJ, USA. Email: adirweesh@stfrancismedical.org

doi: https://doi.org/10.14740/gr860w spread into the mediastinum and extrathoracic locations, the actual incidence of primary DLBCL of the lung may be underestimated. To our knowledge, PPDLBCL has been reported only in case reports or small series [4-6].

\section{Case Report}

A 77-year-old male with past medical history significant for hypertension, hyperlipidemia, gout and osteoarthritis presented with persistent epigastric pain for months. His pain responded quite poorly to pantoprazole, and different pain medications. Overall, he has lost 30 pounds in the last 3 months. He also continued to have occasional blood per rectum. His bowel habits, for the most part, were described as more constipation. He denied any right upper quadrant pain, jaundice or dark urine, nausea, vomiting or difficulty in swallowing. He also denied fever, chills, night sweating, rash, pruritus, easy or unexplained bruising. The rest of the review of systems was otherwise unremarkable. His examination was significant for epigastric tenderness. The rest of the systemic and abdominal examination was grossly normal.

An upper endoscopy performed revealed a mass in the gastric body and antral gastritis (Fig. 1a and b). Multiple gastric biopsies were taken. He also underwent a full colonoscopy examination that showed two polyps in the transverse colon; all were removed by cold biopsy polypectomy, and bleeding rectal angiodysplasia was treated with argon beam coagulation.

Gastric body mass biopsy showed involvement by diffuse large B-cell lymphoma (Fig. 2a, b and c). Studies showed the large cells express CD45, CD20, CD79a, PAX5, BCL-2, MUM-1 and BCL-6.

The patient had a computed tomography (CT) scan of chest, abdomen and pelvis which showed a solitary right lung mass. A CT-guided fine-needle aspiration cytology also showed a DLBCL (Fig. 3a, b and c). He tested negative for $H$. pylori. The patient started chemotherapy with $\mathrm{CHOP}$ regimen and on follow-up with oncology department.

\section{Discussion}

Histopathologically, 90\% of primary GI lymphomas are B- 

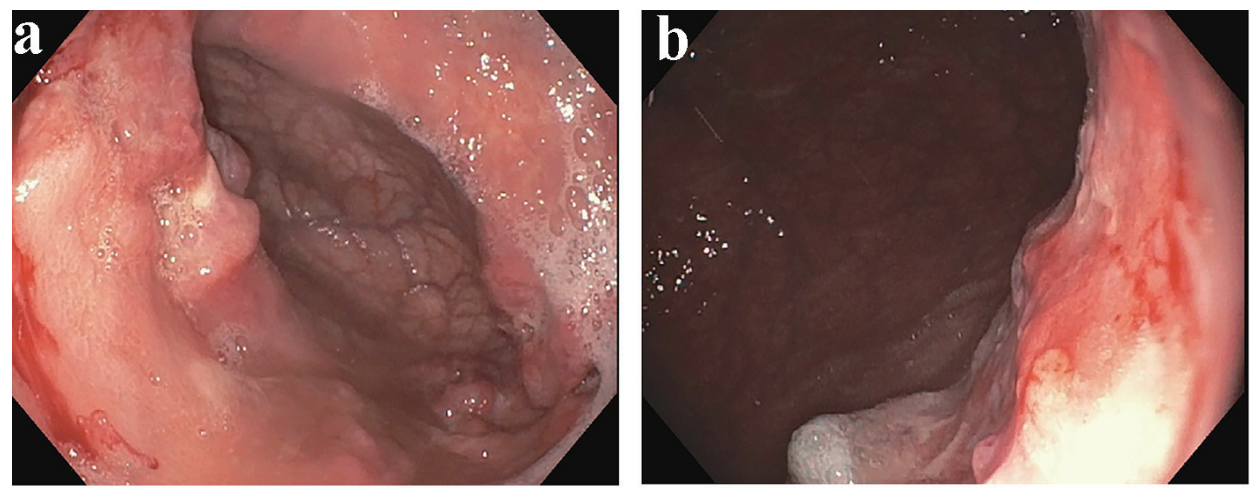

Figure 1. (a, b) A diffuse gastric body mass.

cell origin with T-cell and Hodgkin lymphoma accounting for the remaining $10 \%$ [1]. DLBCL can be divided into three subgroups, namely germinal-center B-cell-like, activated Bcell-like, and primary mediastinal DLBCL [1]. Some of the gene translocations involved includes $\mathrm{t}(14 ; 18)$ (q32;q21) with BCL2-rearrangement, $t(3 ; 14)$ (p27;q32) with BCL6-rearrangement and $\mathrm{t}(8 ; 14)(\mathrm{q} 24 ; \mathrm{q} 32)$ with MYC rearrangement, respectively [7]. Some of the risk factors and predisposing conditions for the development of gastric lymphoma include $H$. pylori infection, immunosuppression and inflammatory bowel disease. Studies have also demonstrated an increased risk of primary GI B-cell lymphoma in patients with celiac disease [8].

Primary gastric lymphoma is indistinguishable from other types of gastric carcinoma by endoscopy alone. The three main endoscopic patterns are non-specific and include ulceration, diffuse infiltration, and polypoid mass [1]. Endoscopy is useful however for obtaining a biopsy and for follow-up. EUS can assess the extent of lesion and its invasion [1]. Diffuse infiltration involving more than $50 \%$ of the stomach and segmental infiltration are the most common features of gastric NHL on CT images [1]. MRI features include irregularly thickened mucosal folds, irregular submucosal infiltration, annular constricting lesion exophytic tumor growth, mesenteric masses and mesenteric/retroperitoneal lymphadenopathy [1].

PPLs usually originate from MALT, either developing into Hodgkin lymphoma or NHL. The most common type of PPL is MALT lymphoma, and DLBCL is a rare form with a poorer prognosis than MALT lymphoma. The incidence of PPL increases with age and peaks in the fifth and seventh decades of life. There is a slightly higher male-to-female ratio [9]. The PPDLBCL usually presents with non-specific respiratory symptoms [10]. The radiological findings are also often nonspecific, which makes it challenging to diagnose. It can lead to the misdiagnosis of inflammation, tuberculosis, even lung cancer. For the definitive diagnosis, the invasive open lung biopsy or CT-guided fine-needle aspiration cytology is required [11]. With its non-specific presentation, the diagnosis of PPDLBCL is very challenging and often leads to misdiagnosis or delayed diagnosis.

The key features of CT are single or multiple solid pulmonary nodules or masses, cavitation and mediastinal lymph node enlargement $[2,5,11]$. Consolidation is rarely found [10]. The reversed halo sign (RHS) proposed by Marchiori et al could be useful for differentiating invasive pulmonary aspergillosis from pulmonary lymphoma [12]. Recently, FDG-PET/CT has emerged as a powerful functional imaging tool. It has high sensitivity and specificity for the diagnosis of lymphoma with or without invasion anywhere in the whole body. It also helps in lymphoma staging and therapy response assessment in lymphoma [13].

According to Coiffier et al, it may beneficial to add rituximab to $\mathrm{CHOP}$ chemotherapy vs. $\mathrm{CHOP}$ chemotherapy alone, for patients with newly diagnosed DLBCL after they found significant increases in the rate of complete response, decrease in the rates of treatment failure and relapse, and improvement in overall survival as compared with standard CHOP alone [14]. Another study compared surgical resection plus chemotherapy versus chemotherapy alone for primary gas-
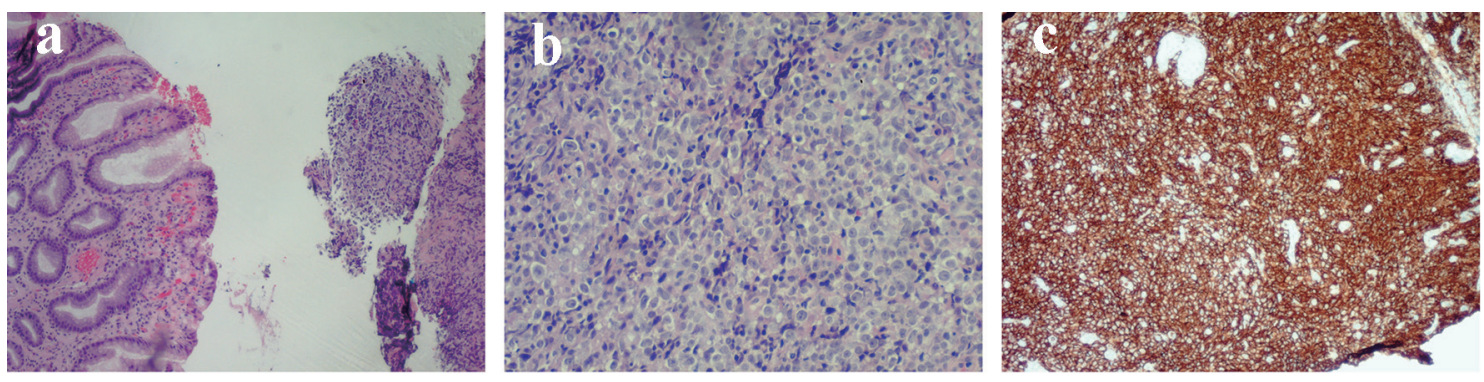

Figure 2. (a) Gastric mucosa with chronic inflammation (left), diffuse infiltrate by large neoplastic cells (right). (b) The large neoplastic cells with prominent nucleoli, irregular contours and vesicular nuclear chromatin. (c) The diffuse staining by CD20 (a lymphocyte marker). 

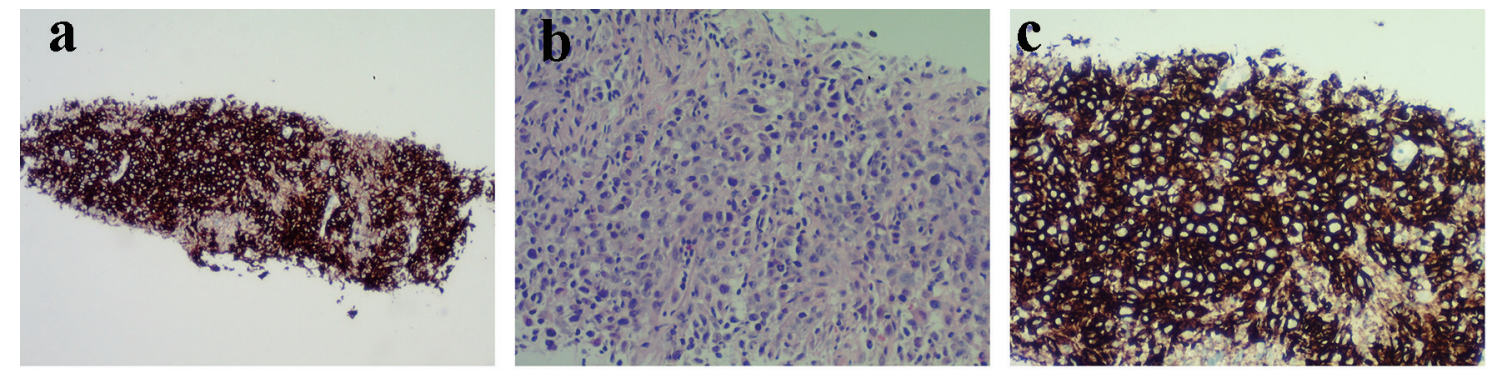

Figure 3. (a) Diffuse infiltrate by neoplastic cells, no lung parenchyma seen. (b) Large neoplastic cells with prominent nucleoli, irregular nuclear contours and vesicular nuclear chromatin (similar to gastric biopsy). (c) CD20 stain: diffuse staining consistent with diffuse large B-cell lymphoma.

tric DLBCL, found similar 5-year survival rates $(>90 \%)$ for localized gastric DLBCL with surgery plus chemotherapy or chemotherapy alone [15]. The stage of the lymphoma and its histopathological type plays a significant role in the choice of treatment and its prognosis. The treatment options for localized disease include surgical resection, chemotherapy, or radiotherapy. The CHOP chemotherapy regimen has been the mainstay of therapy for the PLL [16]. The addition of the antiCD20 mono-clonal antibody rituximab to this chemotherapy dramatically improved the outcomes, resulting in a $16 \% \mathrm{ab}-$ solute improvement in 10-year overall survival in elderly patients $\geq 60$ years of age [17]. Additional trials further demonstrate the benefit of rituximab and establishment of R-CHOP as the standard of care [8]. The median time to death or disease recurrence is reported as 6 years [9].

\section{Grant Support}

None.

\section{Financial Disclosures}

None.

\section{Conflicts of Interest}

None.

\section{References}

1. Ghimire P, Wu GY, Zhu L. Primary gastrointestinal lymphoma. World J Gastroenterol. 2011;17(6):697-707.

2. Pina-Oviedo S, Weissferdt A, Kalhor N, Moran CA. Primary pulmonary lymphomas. Adv Anat Pathol. 2015;22(6):355-375.

3. Zinzani PL, Martelli M, Poletti V, Vitolo U, Gobbi PG, Chisesi T, Barosi G, et al. Practice guidelines for the management of extranodal non-Hodgkin's lymphomas of adult non-immunodeficient patients. Part I: primary lung and mediastinal lymphomas. A project of the Italian so- ciety of hematology, the Italian society of experimental hematology and the Italian group for bone marrow transplantation. Haematologica. 2008;93(9):1364-1371.

4. Neri N, Jesus Nambo M, Aviles A. Diffuse large B-cell lymphoma primary of lung. Hematology. 2011;16(2):110112.

5. Yoshino N, Hirata T, Takeuchi C, Usuda J, Hosone M. A case of primary pulmonary diffuse large B-cell lymphoma diagnosed by transbronchial biopsy. Ann Thorac Cardiovasc Surg. 2015;21(4):396-398.

6. Jiang AG, Gao XY, Lu HY. Diagnosis and management of a patient with primary pulmonary diffuse large B-cell lymphoma: A case report and review of the literature. Exp Ther Med. 2014;8(3):797-800.

7. Ponzoni M, Ferreri AJ, Pruneri G, Pozzi B, Dell'Oro S, Pigni A, Pinotti G, et al. Prognostic value of bcl-6, CD10 and CD38 immunoreactivity in stage I-II gastric lymphomas: identification of a subset of CD10+ large Bcell lymphomas with a favorable outcome. Int J Cancer. 2003;106(2):288-291.

8. Catassi C, Fabiani E, Corrao G, Barbato M, De Renzo A, Carella AM, Gabrielli A, et al. Risk of non-Hodgkin lymphoma in celiac disease. JAMA. 2002;287(11):14131419.

9. Wei S, Li X, Qiu X, Zhao H, Chen G, Chen J, Zhou Q. Primary lung lymphoma involving the superior vena cava. World J Surg Oncol. 2012;10:131.

10. Kim JH, Lee SH, Park J, Kim HY, Lee SI, Park JO, Kim $\mathrm{K}$, et al. Primary pulmonary non-Hodgkin's lymphoma. Jpn J Clin Oncol. 2004;34(9):510-514.

11. Hare SS, Souza CA, Bain G, Seely JM, Frcpc, Gomes MM, Quigley M. The radiological spectrum of pulmonary lymphoproliferative disease. $\mathrm{Br} \mathrm{J}$ Radiol. 2012;85(1015):848-864.

12. Marchiori E, Godoy MC, Zanetti G, Hochhegger B, Rodrigues RS. The reversed halo sign. Another CT finding useful for distinguish invasive pulmonary aspergillosis and pulmonary lymphoma. Eur J Radiol. 2011;79(2):e96-97.

13. Zhou X, Lu K, Geng L, Li X, Jiang Y, Wang X. Utility of PET/CT in the diagnosis and staging of extranodal natural killer/T-cell lymphoma: a systematic review and meta-analysis. Medicine (Baltimore). 2014;93(28):e258.

14. Coiffier B, Lepage E, Briere J, Herbrecht R, Tilly H, Bouabdallah R, Morel P, et al. CHOP chemotherapy plus 
rituximab compared with $\mathrm{CHOP}$ alone in elderly patients with diffuse large-B-cell lymphoma. $\mathrm{N}$ Engl J Med. 2002;346(4):235-242.

15. Binn M, Ruskone-Fourmestraux A, Lepage E, Haioun C, Delmer A, Aegerter P, Lavergne A, et al. Surgical resection plus chemotherapy versus chemotherapy alone: comparison of two strategies to treat diffuse large B-cell gastric lymphoma. Ann Oncol. 2003;14(12):1751-1757.

16. Fisher RI, Gaynor ER, Dahlberg S, Oken MM, Grogan TM, Mize EM, Glick JH, et al. Comparison of a standard regimen (CHOP) with three intensive chemotherapy regimens for advanced non-Hodgkin's lymphoma. N Engl J Med. 1993;328(14):1002-1006.

17. Coiffier B, Thieblemont C, Van Den Neste E, Lepeu G, Plantier I, Castaigne S, Lefort S, et al. Long-term outcome of patients in the LNH-98.5 trial, the first randomized study comparing rituximab-CHOP to standard CHOP chemotherapy in DLBCL patients: a study by the Groupe d'Etudes des Lymphomes de l'Adulte. Blood. 2010;116(12):2040-2045. 